\title{
Dynamic optical manipulation of colloidal systems using a spatial light modulator
}

\author{
VINCENT R. DARIA, RENÉ L. ERIKSEN and \\ JESPER GLÜCKSTAD \\ Optics and Fluid Dynamics Department, Risø National Laboratory, \\ DK-4000 Roskilde, Denmark \\ e-mail: jesper.gluckstad@risoe.dk
}

(Received 11 September 2002; final version 23 October 2002)

\begin{abstract}
A method and mathematical foundation are presented for generating multiple-beam optical tweezers capable of introducing complex trapping beam configurations that enable optical manipulation for a variety of colloidal structures. The method is based on the generalized phase contrast technique for generating high intensity beam patterns from an input phase modulation encoded on a spatial light modulator. The mathematical foundation describes issues concerning how the method provides high photon efficiency adequate for generating large array traps while maintaining dynamic features. Experimental results show multiple trapping of up to 25 particles using a $200 \mathrm{~mW}$ laser diode operating at $830 \mathrm{~nm}$. Arbitrary array beam configurations are also shown where the shape, position and size can easily be reconfigured and applied for dynamic manipulation of colloidal particles.
\end{abstract}

\section{Introduction}

Observation and manipulation of colloidal structures in micro- and nano-scale has attracted considerable attention because it can provide answers to fundamental issues on the forces that constitute arrays of particles and verify theories on selfassembly of materials, particularly of crystalline structures [1]. The study of these structures also enables development of practical applications that are based on large array systems for use in photonic crystals, micro-fluidic processes and biochemical sensing devices [2, 3]. This can, in time, be the future of nano-scale hybrid processing systems and a prelude to photonic computing devices and sensing chips.

Current techniques used in the study of colloidal structures, however, entail only passive observation methods, such as the use of image forming instruments based on optical microscopes or scanning probe techniques. Similarly, in cases where aggregated structures are fabricated, the methods involved, either by self- or guided-assembly, are hampered by the lack of controllability that results in structural arrays that are both spatially and temporally inflexible. In order to achieve a comprehensive study of colloidal structures, it is desirable to develop techniques that enable active observation as well as dynamic formation and manipulation of arbitrary arrays of particles. A single element within a colloidal structure has been demonstrated to be trapped and manipulated by means of 
optical tweezers $[4,5]$, which is now an established tool for manipulation of individual particles. Single beam optical tweezers, however, fall short when the aim is to achieve parallel and simultaneous trapping of a plurality of particles. There have been many schemes to extend conventional optical tweezer systems to facilitate multiple trapping using high-speed scanning mirrors [6-8] or multiple light sources [9]. These systems operate efficiently for a limited number of traps, but intensify in complexity or degrade in performance as the number of beams is increased to simultaneously trap a large number of particles. Hence, a desirable system for dynamic and simultaneous manipulation of colloids is a multiple-beam optical tweezer (MOT) system that is based on a simple yet efficient method that enables re-distribution and arbitrary shaping of a collimated incident laser beam.

Generation of MOT based on computer-generated holograms (CGH) for trapping an array of particles has been demonstrated [10,11]. In this method, a phase-grating pattern, or kinoform, is encoded on a phase-only spatial light modulator (SLM) to distribute a collimated incident beam into a range of angles that are directed to the back aperture of an objective lens, subsequently forming the desired tweezer-beam configuration at the spatial Fourier plane. Encoding a grating-based pattern on an SLM, however, gives limited performance due to the restricted space-bandwidth product (SBWP) and the modulation transfer function (MTF) that reveals low diffraction efficiency of the SLM [12]. The high spatial frequencies of a kinoform are therefore not encoded completely on an SLM resulting in a limited tweezer-beam configuration and strongly restricted movements of a plurality of beams at the output. For efficient operation on large particle arrays, it is therefore reasonable to compensate this inefficiency either by constricting the tweezer beams to a symmetric configuration reconstructed around the zero-order beam, or by increasing the input laser power to an amount that is possibly beyond the power tolerance of SLMs. It is worthwhile to note, however, that the CGH approach works efficiently for a fixed MOT such that kinoforms are pre-fabricated in an optical element by means of high-resolution lithographic recording [13].

Despite the low SBWP and diffraction efficiencies of phase-only SLMs, it is still an efficient device for achieving a fully dynamic MOT by applying an imaging operation and using the generalized phase contrast (GPC) method [14-16]. In this method, generation and dynamic manipulation of multiple beams are achieved by an imaging operation of an input phase pattern that is directly mapped to the output tweezer-plane and is therefore not seriously affected by the limited SBWP and MTF of the SLM. The GPC-based MOT does not depend on complex iterative algorithms, hence simultaneous manipulation of particles can be performed in real time and moved with a speed that is only limited by the response time of the SLM. Even though this technique has been successful in demonstrating real-time dynamic manipulation of particles, its potential operation to colloidal structures and large array systems has yet to be studied and emphasized.

In this work, the GPC-MOT and an SLM are used to demonstrate complex trapping beam configurations that enable optical manipulation of a variety of colloidal structures. The GPC method is discussed briefly and the mathematical foundation describing issues concerning how the method provides high photon 
efficiency adequate for generating large array traps while maintaining dynamic features is detailed. An experimental setup for trapping and dynamic manipulation of multiple polystyrene particles is described. Arbitrary and large array beam configurations are demonstrated in which the shape, position and size can easily be reconfigured, depending on the desired application.

\section{The generalized phase contrast method}

The theoretical basis for the GPC-based MOT has been developed from an analysis of a phase-only optical projection system [17], which is a full generalization of the Zernike phase contrast scheme [18]. Using this approach, the small phase angle limitation of Zernike's method is no longer a limiting constraint and the complete range of phase values from zero to $2 \pi$ can be utilized. It has been shown that a system in which the spatial average value of the input phase modulated light is carefully matched to the phase shifting value of a phase contrast filter can produce an essentially lossless phase-to-intensity mapping of the input light $[17,19]$.

The setup considered is based on the spatial filtering architecture illustrated in figure 1. An output intensity distribution produced from an input phase pattern is obtained by applying a truncated, on-axis, phase-filtering operation in the spatial frequency domain between two Fourier transforming lenses. The first lens performs a spatial Fourier transform, so that direct propagated light is focused onto this on-axis filtering region, whereas spatially varying phase information generates light scattered to locations outside this central region. By applying a phase difference between the two filtering regions, one can obtain an intensity pattern at the observation plane using the second Fourier lens. The GPC method works optimally in cases where there is a large separation between the on-axis, low spatial frequency light and the higher spatial frequencies in the Fourier plane. The pseudo-periodicity inherent in colloidal particle arrays provides the good separation of high and low spatial frequencies required for optimal performance of this system.

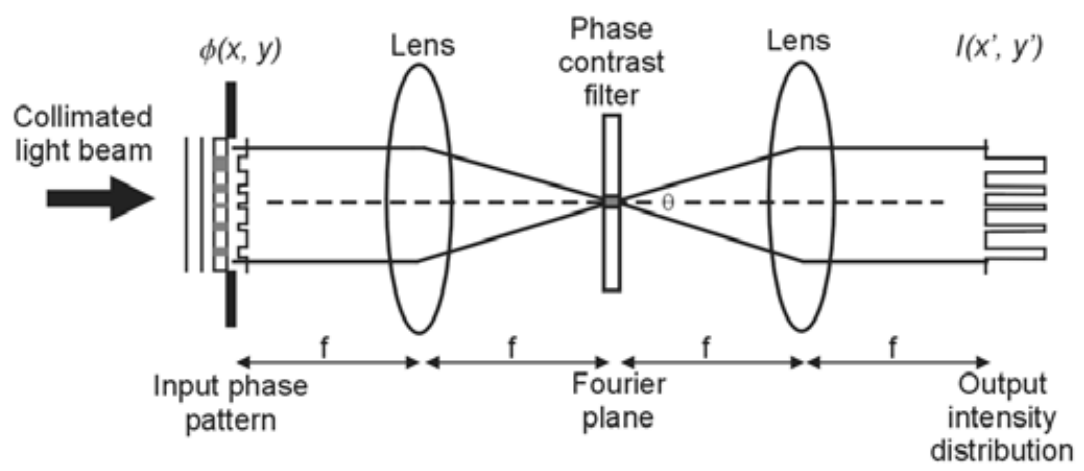

Figure 1. Spatial filtering architecture based on a $4 f$ optical system for lossless conversion of an input phase pattern $\phi(x, y)$ to an output intensity distribution $I\left(x^{\prime}, y^{\prime}\right)$. The phase contrast filter at the Fourier plane sets the phase shift $\theta$ of the low frequency spatial component of an incident wavefront. 


\section{Mathematical analysis}

The first step in the analysis is derivation of a relationship between the input phase values and the output intensity. This approach is based directly on detailed derivations previously obtained for the $\operatorname{GPC} \operatorname{method}[17,19,20]$.

One can write an expression for the intensity at the observation plane $\left(x^{\prime}, y^{\prime}\right)$ of the setup shown in figure 1 , such that:

$$
I\left(x^{\prime}, y^{\prime}\right)=\left|\exp \left(\mathrm{i} \tilde{\phi}\left(x^{\prime}, y^{\prime}\right)\right) \operatorname{circ}\left(r^{\prime} / \Delta r\right)+g\left(r^{\prime}\right)\right| \bar{\alpha}|(\exp (\mathrm{i} \theta)-1)|^{2}
$$

with

$$
\left\{\begin{array}{l}
\bar{\alpha}=A^{-1} \iint_{A} \exp (\mathrm{i} \phi(x, y)) \mathrm{d} x \mathrm{~d} y=|\bar{\alpha}| \exp \left(\mathrm{i} \phi_{\bar{\alpha}}\right) \\
\tilde{\phi}\left(x^{\prime}, y^{\prime}\right)=\phi\left(x^{\prime}, y^{\prime}\right)-\phi_{\bar{\alpha}} \\
r^{\prime}=\sqrt{x^{\prime 2}+y^{\prime 2}} .
\end{array}\right.
$$

In this formulation, $\bar{\alpha}$ is the spatial average of the input wavefront and as such is generally complex with absolute value $|\bar{\alpha}|$ and phase $\phi_{\bar{\alpha}}$. The input phase pattern, $\phi(x, y)$, is addressed on the phase-only SLM with a circular truncated aperture area $A=\pi(\Delta r)^{2}$. Equation (1) also includes the parameters $\theta$ for the zero-order phase shift of the filter and $g\left(r^{\prime}\right)$ describes the spatial profile of the synthetic reference wave (SRW), diffracted from the aperture formed by the on-axis centred filtering region. It is the interference between this SRW term, carrying information about the filtering parameters, and the imaged phase object that generates the output intensity pattern. It is important to obtain a precise description for the SRW and thereby an accurate interpretation of equation (1).

For a circular input aperture with radius $\Delta r$ we can describe the radius of the corresponding central phase shifting region of the Fourier filter (characterized by the parameter $\theta$ ) in terms of a radial spatial frequency range $\Delta f_{r}$. We obtain the following expression for the SRW by use of the zero-order Hankel transform:

$$
g\left(r^{\prime}\right)=2 \pi \Delta r \int_{0}^{\Delta f_{r}} J_{1}\left(2 \pi \Delta r f_{r}\right) J_{0}\left(2 \pi r^{\prime} f_{r}\right) \mathrm{d} f_{r} .
$$

In order to simplify the analysis, we introduce a term $\eta$, which explicitly relates the radius of the central filtering region to the radius of the main lobe of the Airy function resulting from the Fourier transform of the circular input aperture alone. We can thus express $\eta$ in terms of $\Delta r$ and $\Delta f_{r}$ such that:

$$
\eta=(0.61)^{-1} \Delta r \Delta f_{r}
$$

where the factor 0.61 arises from the radial distance to the first zero crossing of the Airy function corresponding to half of the Airy main lobe factor, 1.22. If we make this substitution in equation (3) and then perform a series expansion in $r^{\prime}$, we obtain the following expression for the SRW:

$$
\begin{aligned}
g\left(r^{\prime}\right)= & 1-J_{0}(1.22 \pi \eta)-\left[(0.61 \pi \eta)^{2} J_{2}(1.22 \pi \eta)\right]\left(r^{\prime} / \Delta r\right)^{2} \\
& +\left\{\left[(0.61 \pi \eta)^{3} / 4\right]\left[2 J_{3}(1.22 \pi \eta)-0.61 \pi \eta J_{4}(1.22 \pi \eta)\right]\right\}\left(r^{\prime} / \Delta r\right)^{4} \ldots
\end{aligned}
$$


In this expansion, the SRW is expressed in radial coordinates normalized to the radius of the imaged input aperture. This can easily be scaled to allow for magnification within the imaging system, although for the remainder of this analysis a direct imaging operation is assumed. From equation (5) it is apparent that the SRW will change as a function of the radius of the central filtering region. Additionally, it is clear that the SRW profile is not necessarily flat over the system output aperture.

To generate large MOT arrays the value of $\eta$ can, in most cases, be chosen to completely encompass the zero-order light with the result that the constant, spaceinvariant term $K=g\left(r^{\prime}=0\right)$ tends to unity as the Bessel function tends to zero. For this particular case, the SRW becomes a flat top profile where we can achieve nearly $100 \%$ light efficiency. For smaller and irregular arrays it will be shown that fine-tuning $\eta$ in the region $0.4-0.6$ provides for an efficient operating regime while maintaining minimal losses.

Using the fact that we wish to generate a dark background for MOT arrays at the observation plane as our principal design criterion, we can write:

$$
I\left(x_{0}^{\prime}, y_{0}^{\prime} ; \tilde{\phi}_{0}\right)=0
$$

where $\left(x_{0}^{\prime}, y_{0}^{\prime}\right)$ indicates observation plane coordinates of the background of the array illumination and $\tilde{\phi}_{0}$ is the phase shift generating a zero-intensity level at the observation plane.

Applying the dark background condition of equation (6) to equation (1) we obtain the following expression:

$$
K|\bar{\alpha}|(1-\exp (\mathrm{i} \theta))=\exp \left(\mathrm{i} \tilde{\phi}_{0}\right)
$$

where we have assumed that the influence of the SRW is sufficiently described by the constant, space invariant term $K=g\left(r^{\prime}=0\right)$.

A key point arising from equation (7) is that we now have a simple way of expressing a new design criterion relating the spatial average value of any input phase pattern to the zero-order phase shift of a matched Fourier phase filter. Since $K$ is, by definition, positive, and by taking the modulus of equation (7) we obtain:

$$
K|\bar{\alpha}|=|2 \sin (\theta / 2)|^{-1} .
$$

Equation (8) makes it possible to deduce the range of valid phase parameters fulfilling the design criteria equation (6). The largest possible absolute value that the complex spatial average value $\bar{\alpha}$ can have is unity; this leads to the following solution interval for equation (8) within the first $2 \pi$ phase-cycle:

$$
\theta=[\pi / 3 ; 5 \pi / 3]
$$

From equation (8) we also observe that $K|\bar{\alpha}|$ can take on a value limited to the interval:

$$
K|\bar{\alpha}|=[1 / 2 ; 1]
$$


Equation (8) and the solution intervals described by equations (9) and (10) specify the design parameters for achieving optimal performance in extracting and displaying the phase information carried by the incoming wavefront. Moreover, equation (8) hints at extra means of optimization by encoding the phase modulation depth on the SLM itself in addition to the filter parameter. This last approach is particularly useful when the filter parameter has a restricted dynamic range or is fixed.

Now, assuming a fixed filter, the best choice for the filter parameter is a value that allows for the largest dynamic range of phasor values at the input. Accordingly, the smallest possible real value, $K \bar{\alpha}=1 / 2$, is desirable implying that $\theta=\pi$, leading to the output intensity distribution:

$$
I\left(x^{\prime}, y^{\prime}\right)=2\left[1-\cos \left(\phi\left(x^{\prime}, y^{\prime}\right)\right)\right]
$$

Inserting $K \bar{\alpha}=1 / 2$ in equation (2), we obtain the following two requirements for the SLM phase function $\phi(x, y)$ :

$$
\left\{\begin{array}{l}
K A^{-1} \iint_{A} \cos (\phi(x, y)) \mathrm{d} x \mathrm{~d} y=\frac{1}{2} \\
K A^{-1} \iint_{A} \sin (\phi(x, y)) \mathrm{d} x \mathrm{~d} y=0
\end{array}\right.
$$

Observe that it is only the first requirement in equation (12) that is directly related to the output intensity in equation (11) via the cosine term. Since there are always two choices for a given phasor value that result in the same cosine value (excluding 0 and $\pi$ ), we notice that the second requirement can subsequently be fulfilled independently of the first requirement simply by complex conjugating an appropriate number of phasor values. This fact is a major point in the GPC method since it makes it possible to solely concentrate on the first requirement in the process of synthesizing a desired intensity pattern.

In most cases, we are interested only in equalized output intensity levels for the tweezer-beams in the array since the smooth transitions required for lateral trapping are almost inevitably achieved by the finite spatial bandwidth of the train of optical components (inherent blurring of sharp edges). In the succeeding analysis, we therefore focus on the encoding of SLM phase levels to achieve binary output intensity levels. Previously, it was shown [21] that a derivation based on ternary phase levels allows for the widest range of binary intensity pattern encodings. In addition, the ternary phase analysis automatically provides the simplified but very important binary phase level encoding as a special case.

For ternary phase encoding, consider the illuminated portion of the SLM aperture area $A$ to be divided into sub-areas $A_{0}, A_{1}$ and $A_{2}$ with respective phase values $\phi_{0}, \phi_{1}$ and $\phi_{2}$. We are aiming for the derivation of general expressions relating the addressing parameters for the SLM to the range of possible phase parameters of the Fourier filter obeying the design criterion already set out.

The total area defined by the truncated area of the SLM and its average phase modulation can be expressed as the sum of the phase-weighted sub-areas:

$$
A_{0} \exp \left(\mathrm{i} \tilde{\phi}_{0}\right)+A_{1} \exp \left(\mathrm{i} \tilde{\phi}_{1}\right)+A_{2} \exp \left(\mathrm{i} \tilde{\phi}_{2}\right)=A|\bar{\alpha}| \text {. }
$$


This can be further simplified by expressing the sub-areas as fractions of the total area $A$ such that $F_{1}=A_{1} / A$ and $F_{2}=A_{2} / A$ :

$$
\left(1-F_{1}-F_{2}\right) \exp \left(\mathrm{i} \tilde{\phi}_{0}\right)+F_{1} \exp \left(\mathrm{i} \tilde{\phi}_{1}\right)+F_{2} \exp \left(\mathrm{i} \tilde{\phi}_{2}\right)=|\bar{\alpha}| \text {. }
$$

For the MOT patterns considered here, we are interested in binary intensity patterns with levels corresponding to the input phase values. In this case the dark background region is defined by $\left(A_{0}, \tilde{\phi}_{0}\right)$ and the bright output level of intensity $I$ is determined by $\left(A_{1}, \tilde{\phi}_{1}\right)$ and $\left(A_{2}, \tilde{\phi}_{2}\right)$ in the input plane. For the binary output intensity condition it follows that:

$$
I\left(\tilde{\phi}_{1}\right)=I\left(\tilde{\phi}_{2}\right)
$$

This equality corresponds to a symmetric condition explained previously by use of phasor chart analysis [17]. Due to this symmetry, one can simplify the analysis by applying the following substitution:

$$
\Delta \phi=\tilde{\phi}_{1}-\tilde{\phi}_{0}=\tilde{\phi}_{0}-\tilde{\phi}_{2}
$$

so that equation (14) can be rewritten as

$$
F_{1}(\exp (\mathrm{i} \Delta \phi)-1)+F_{2}(\exp (-\mathrm{i} \Delta \phi)-1)=K^{-1}(1-\exp (\mathrm{i} \theta))^{-1}-1 .
$$

It is now a straightforward task to solve equation (17) for the real part and the imaginary part respectively, to obtain the following sets of equations:

$$
\left\{\begin{array}{l}
F_{1}+F_{2}=(2 K-1)(2 K(1-\cos (\Delta \phi)))^{-1} \\
F_{1}-F_{2}=\sin (\theta)(2 K \sin (\Delta \phi)(1-\cos (\theta)))^{-1}
\end{array}\right.
$$

This can also be expressed in terms of the fractional areas, such that:

$$
\left\{\begin{array}{l}
F_{1}=(4 K)^{-1}\left[(2 K-1)(1-\cos (\Delta \phi))^{-1}+\sin (\theta)(\sin (\Delta \phi)(1-\cos (\theta)))^{-1}\right] \\
F_{2}=(4 K)^{-1}\left[(2 K-1)(1-\cos (\Delta \phi))^{-1}-\sin (\theta)(\sin (\Delta \phi)(1-\cos (\theta)))^{-1}\right] .
\end{array}\right.
$$

Since the focus has been on solutions where identical intensity levels are obtained in both the $F_{1}$-region and the $F_{2}$-region, one can define the MOT array compression factor $C$ in the following way:

$$
C=\left(F_{1}+F_{2}\right)^{-1}=\left(1-(2 K)^{-1}\right)^{-1}(1-\cos (\Delta \phi)) .
$$

The minimum compression factor corresponds to uniform illumination at the output such that $F_{1}+F_{2}=1$, whereas the maximum compression factor is found from equation (20) to be $C \rightarrow \infty$ for $K=1 / 2$ (but less light efficient due to increased scattered light outside the working region [17]).

An interesting special case can be deduced from equation (17) by setting $F_{2}=0$, where we find that:

$$
F=F_{1}=(K(1-\exp (\mathrm{i} \theta))-1)(K(1-\exp (\mathrm{i} \Delta \phi))(1-\exp (\mathrm{i} \theta)))^{-1},
$$


implying that for the binary phase modulation case we must have:

$$
\Delta \phi=\theta
$$

in order for $F$ to be real valued.

This result turns out to be the special case that corresponds to the set of solutions where a binary phase pattern serves as the input. In the next section we illustrate how this set of solutions provides for efficient MOT encoding.

In a practical reconfigurable and binary modulated MOT system it should be possible for the user to add and remove beams depending on the specific application without affecting the trapping strength significantly. Changing the number and/or the size of each beam results in a change in the fraction of modulated light with the fill factor $F$. By calculating the visibility of each trapping beam as a function of $F$, it is possible to have a rough measure of how much the efficiency of each trap varies as a function of this parameter.

The ratio of the radius of the PCF to that of the spatial frequency distribution describing the focused light spot at the Fourier plane strongly influences the output contrast. We previously analysed the term $K$, which explicitly relates the radius of the central filtering region to the radius of the main lobe of the Airy function resulting from a Fourier transform of the circular input aperture. Based on this analysis the following expression is obtained for the visibility $V$, that depends on only $K$ and $F$ [17]:

$$
V=4\left|1-2 F_{\pi}\right| K\left(1+4 K^{2}\left(1-2 F_{\pi}\right)^{2}\right)^{-1}
$$

where $F_{\pi}$ indicates the desirable choice of using $0 / \pi$ for the binary SLM modulation and $\pi$ for a matched filter as invoked by equation (22).

Figure 2 shows a plot of the visibility as a function of the fractional area of $\pi$ modulation $F_{\pi}$ for the range 0 to 0.5 . Three curves are plotted for the values of $\eta=0.4, \eta=0.5$ and $\eta=0.6$. Typically the MOT beams would occupy a fractional area less than 0.2 because there is a physical upper limit for how close and dense the tweezer-beams can be placed in order to give sufficient separation of the trapped particles. Studying figure 2 it is possible to see that a value $\eta=0.5$ ensures nearly constant visibility for $F_{\pi}<0.2$.

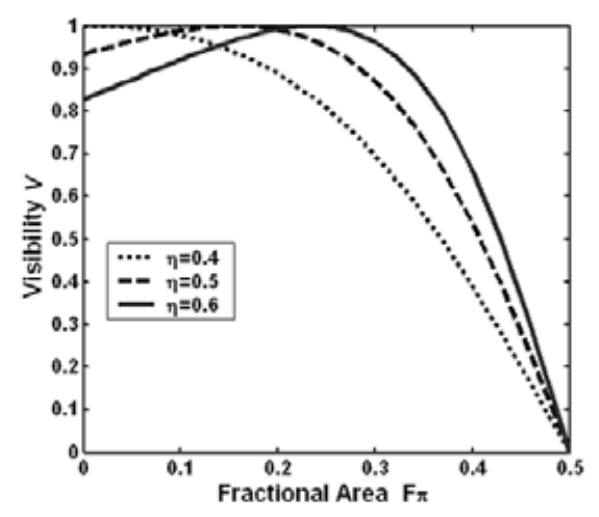

Figure 2. Plot of the visibility as a function of the fractional area of $\pi$ modulation $F_{\pi}$ for the range 0 to 0.5 and for values $\eta=0.4, \eta=0.5$ and $\eta=0.6$. 


\section{Experiments}

This section presents experimental results demonstrating the versatility of the GPC-MOT method. Results are presented showing different trapping experiments and proposed intensity patterns with arbitrary number, shape and size that are useful for the manipulation of colloidal structures. Due to currently very limited laser diode power levels, actual manipulation of large arrays of particles is unattainable. We have therefore focused on conceptually verifying the functionality of this system and its usefulness within colloidal studies based on small-array 'proof of principle' experiments.

The experimental set-up for the GPC-MOT is shown in figure 3 . The expanded and collimated laser beam from a $200 \mathrm{~mW}$ diode laser $(\lambda=830 \mathrm{~nm})$ is incident on a reflection-geometry phase-only SLM. The SLM is a parallel-aligned nematic liquid crystal type (Hamamatsu Photonics), which is optically addressed by a VGA-resolution $(640 \times 480$ pixels $)$ liquid crystal projector element that is controlled from the video output of a computer (PC). A $6 \mathrm{~mm}$ diameter iris (Ir) is placed in front of the SLM. The diameter of the iris is matched to the diameter of the phase contrast filter (PCF) in order to optimize the contrast of the output intensity distribution [17]. The phase-modulated light is directed into the $4 f$ filtering system that is composed of lenses L1 and L2, and a $50 \mu \mathrm{m}$ diameter PCF (fixed $\pi$ phase shift) positioned at the Fourier plane. A high-contrast intensity distribution, which is directly related to the phase pattern on the SLM, is generated in the image plane (IP), which also corresponds to the back focal plane of L2. It is possible to monitor the output intensity distribution by placing a CCD camera in this plane (IP). The lens L3 and the microscope objective, MO, form the second $4 f$ lens system that scales the intensity distribution in the tweezer-plane. The fluorescence port of an inverted microscope (Leica DM-IRB) is used to couple the infrared laser light to the back focal plane of the MO via a dichroic mirror (DM). The trapped particles are imaged in a CCD camera using the same $\mathrm{MO}$ and the tube lens, TL. The total de-magnification of the modulation aperture of the SLM is $1 / 167$. This results in an effective $36 \mu \mathrm{m}$ diameter working region in the tweezer-plane. The size of one resolution cell (pixel) at the SLM is $41.4 \mu \mathrm{m}$ [12], corresponding to $0.25 \mu \mathrm{m}$ in the tweezer-plane, which therefore is the smallest incremental movement of a trapping beam for the current configuration.

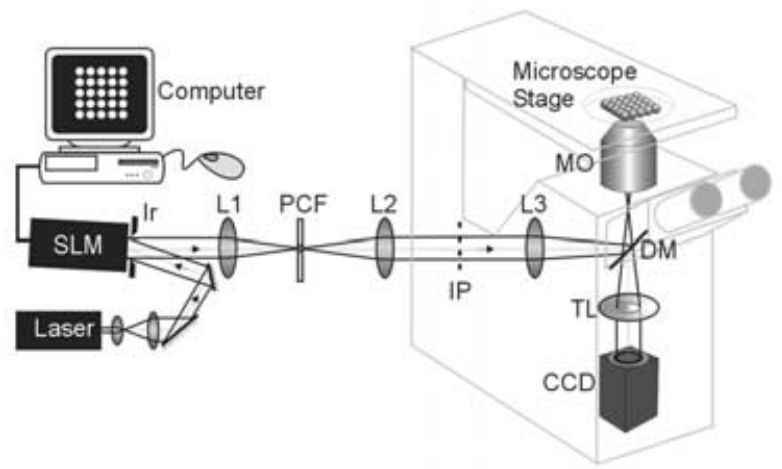

Figure 3. Schematic diagram of the experimental setup for implementing dynamic multiple-beam optical tweezers based on the generalized phase contrast method and a reflection geometry spatial light modulator. 


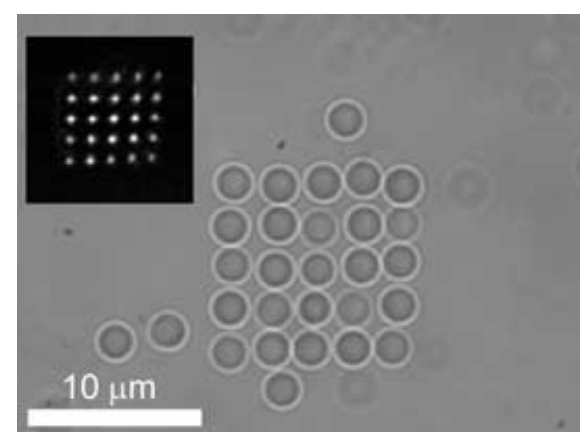

Figure 4. Optical trapping of 25 silica beads, $2.25 \mu \mathrm{m}$ in diameter, arranged in a five by five array. The spacing between each bead is $3 \mu \mathrm{m}$. The inset at the upper left corner shows the measured GPC generated intensity distribution in the tweezer-plane.

After appropriate calibration of the SLM, the binary gray levels 1 and 150 correspond to the phase shift values 0 and $\pi$, respectively. The diameter of each phase dot at the SLM is 7 pixels, corresponding to a diameter of $1.7 \mu \mathrm{m}$ in the tweezer-plane, which is relatively large, but is found to function satisfactorily with the size of the used beads (bead diameter $\geq$ beam diameter).

The limiting factor for the generation and dynamic manipulation of the tweezer-beams is the response time of the liquid crystals in the SLM. The response time is approximately $100 \mathrm{~ms}$, which corresponds to 10 incremental steps of $0.25 \mu \mathrm{m}$ per second, giving a maximum translation speed of $2.5 \mu \mathrm{m} \mathrm{s}^{-1}$ for this particular setup.

To test the trapping functionality of the system, suspensions of $2 \mu \mathrm{m}$ diameter polystyrene beads and $2.25 \mu \mathrm{m}$ diameter silica beads diluted in de-ionized water were used with a small amount of surfactant added. Figure 4 shows the simultaneous trapping of 25 silica micro-spheres using the GPC-MOT. The inset of the figure shows the measured intensity distribution of the trapping beams in the tweezer-plane. It is worthwhile to note that this trapping beam array does not contain a disturbing zero-order beam in the centre as well as noise from higher-order diffracted beams. In order to have sufficient trapping strength, the laser power for each trap in the tweezer-plane was adjusted between $0.1 \mathrm{~mW}$ and $0.5 \mathrm{~mW}$ limited by the available laser power. The estimated efficiency of the system is $35 \%$, which is mainly due to losses encountered by the use of inappropriate anti-reflection coatings for the objective lens and other optical components at the current operating wavelength of $830 \mathrm{~nm}$ [14].

Figure 5 shows an image sequence that demonstrates the dynamic trapping of nine polystyrene beads $(2 \mu \mathrm{m}$ in diameter) arranged in a $3 \times 3$ array with interparticle spacing of $8 \mu \mathrm{m}$. The middle row containing three particles is simultaneously translated with a constant speed of $1.5 \mu \mathrm{m} \mathrm{s}^{-1}$. The time interval between each image frame is $2 \mathrm{~s}$, as shown in the upper right corner of each frame. The first and sixth frame of the sequence include an arrow indicating the displacement directions of the particles. As can be seen in the sequence, particles present at the bottom left corner of each frame are moving freely due to Brownian motions and are clearly not under the influence of radiation pressure effects from the trapping beams. The laser power of each trap is adjusted so that the particles do not escape the traps during the translation. It is found that at a power 


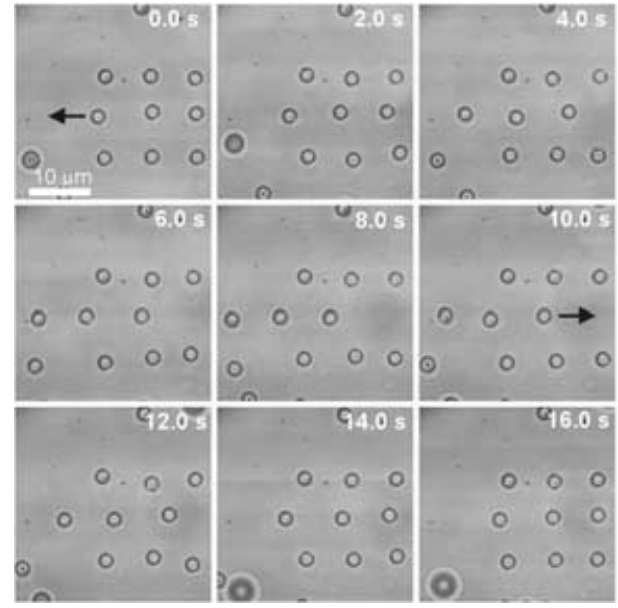

Figure 5. Image sequence showing the dynamic linear displacement of three polystyrene beads, $2 \mu \mathrm{m}$ in diameter, within a three by three array. The three moving beads are linear displaced by $12 \mu \mathrm{m}$ in $8 \mathrm{~s}$, while the other six beads are trapped in fixed positions.

level of $\sim 0.2 \mathrm{~mW}$ the particles begin to escape from the traps, and by using the escape force method [5], assuming laminar flow around the particle, the maximum trapping force for this speed and particle size is calculated as $F_{\text {Trap }}=0.03 \mathrm{pN}$. By doubling the laser power to $\sim 0.4 \mathrm{~mW}$ the particles do not escape and are well confined within the trap. By assuming a harmonic potential, the maximum trapping force at this power level is now doubled to around $F_{\text {Trap }}=0.06 \mathrm{pN}$, which is therefore the expected trapping force for this experiment. In order to minimize the Brownian motions of trapped particles, the laser power should be further increased but as previously explained this is not possible with the available light source of the current setup.

The current setup effectively provides for two-dimensional trapping, which compels the particles to be trapped against the microscope slide. The trapping beams have a relatively collimated intensity distribution along the optical axis, primarily due to the choice of optical components, which was chosen to simplify interfacing with the fluorescence port of a commercially available microscope system. Changing the optical components to make use of the full numerical aperture of the lenses facilitates improved trapping along a single plane with the particles suspended along the optical axis. Moreover, if multiple particles have to be trapped in multiple positions along the optical axis, independent threedimensional manipulation can be introduced in the system by insertion of an additional phase-only SLM for encoding dynamic Fresnel lens functionality for each trapping beam.

Various patterns are proposed for colloidal manipulation of large arrays of particles. In order to achieve large, high-quality trapping arrays, the SLM was modified to be addressed by extended video graphics array (XGA) resolution with $768 \times 768$ pixels, corresponding to a pixel size of $26 \mu \mathrm{m}$. Images were taken at the image plane (IP) of the $4 f$ lens segment of the experimental setup. Figure $6(a)$ shows an array of $15 \times 15$ traps that are periodically spaced. Each spot has a diameter of 6 pixels. For a fixed PCF size of $50 \mu \mathrm{m}$, the aperture size is adjusted to 

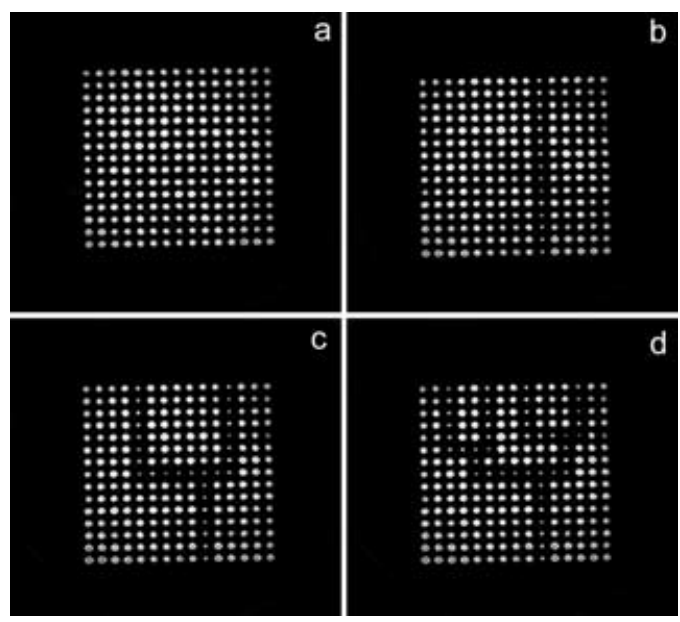

Figure 6. For an input ratio of the input aperture with the PCF diameter of $\eta \cong 0.5$, various trapping beam configurations are demonstrated showing: $(a)$ an array of $15 \times 15$ traps that are periodically spaced with each spot having a diameter of 6 pixels and $F_{\pi} \cong 0.11$; modified trapping beams with certain spots changed to a smaller diameter of 4 pixels corresponding to fractional areas of $(b) F_{\pi} \cong 0.106,(c) F_{\pi} \cong 0.102$ and $(d) F_{\pi} \cong 0.098$.

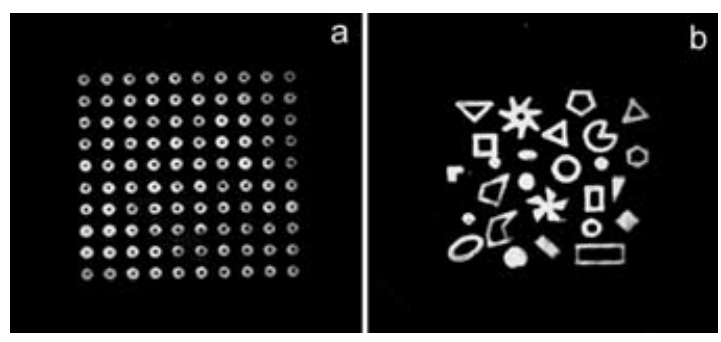

Figure 7. (a) $10 \times 10$ array of optical doughnut beams with estimated fractional area $F \cong$ 0.134. (b) Demonstrating the potential of the GPC-MOT to generate arbitrary geometries and sizes of multiple tweezer-beams by using graphical software to encode any desired pattern on the phase-only SLM.

obtain maximum contrast, with each spot having approximately four times the intensity of the incident light source. Maximum visibility for this pattern is achieved with a fractional area of $\pi$ modulation estimated to be $F_{\pi} \cong 0.11$ and the ratio of the Fourier transformed input aperture with the PCF diameter $\eta \cong 0.5$. Figures $6(b)$ to $6(d)$ show modified arrays with certain spots changed to a smaller diameter of 4 pixels, corresponding to fractional areas of $F_{\pi} \cong 0.106,0.102$ and 0.098, respectively. Despite the modification, the output intensity corresponding to the strength of the trapping beams is not affected, which is shown in figure 2, where the visibility is nearly constant around $F_{\pi} \cong 0.1$ for $\eta \cong 0.5$. Figure $7(a)$ shows a $10 \times 10$ array of optical doughnut beams, which are generated without going through the interference of Laguerre-Gaussian modes [22]. Optical doughnut beams are useful for trapping particles having lower index of refraction than the surrounding medium [23]. The estimated fractional area of the trapping configuration shown in figure $7(a)$ is 0.134 . Figure $7(b)$, on the other hand, 
demonstrates the potential of the GPC-MOT to generate arbitrary geometries and sizes of multiple-tweezer beams by using graphical software to encode desired patterns on the phase-only SLM. Irregular or rectangular beam formations have been used for dynamic rotation of an elongated particle [24].

\section{Conclusions}

The potential of the GPC-based multiple-beam optical tweezers to generate complex trapping beam configurations that enable optical manipulation of a variety of colloidal structures has been demonstrated. The mathematical foundation of the GPC method describing issues concerning how the method provides high photon efficiency adequate for generating large array traps while maintaining dynamic features has been demonstrated. Within the limited power condition of $200 \mathrm{~mW}$, up to 25 particles have been trapped. Fully dynamic movement of multiple particles in an array has also been demonstrated. Finally, arbitrary array beam configurations where the shape, position and size can easily be reconfigured and applied to dynamic manipulation of colloidal systems have been shown. Simultaneous and dynamic optical manipulation of a large number of particles opens up a wide range of applications, such as in guided assembly of photonic crystals, micro-fluidic processes, all-optical control of lab-on-a-chip devices, photonic computing devices and biochemical sensing devices.

\section{Acknowledgments}

This work is supported by the Danish Technical Scientific Research Council. We express our gratitude to T. Hara and Y. Kobayashi of Hamamatsu Photonics for useful discussions on the operation of the SLM.

\section{References}

[1] Velev, O., Lenhoff, A., and Kaler, E., 2000, Science, 287, 2240.

[2] Terray, A., Oakley, J., and Marr, D., 2002, Science, 296, 1841.

[3] Hayward, R., Saville, D., and Aksay, A., 2000, Nature, 404, 56.

[4] Ashrin, A., Dziedic, J. M., Bjorkholm, J. E., and Chu, S., 1986, Opt. Lett., 11, 288.

[5] Svoboda, K., and Block, S. M., 1994, Annu. Rev. Biophys. Biomol. Struct., 23, 247.

[6] Sasaki, K., Koshioka, M., Misawa, H., Kitamura, N., and Masuhara, H., 1991, Opt. Lett., 16, 1463.

[7] Fällman, E., and Axner, O., 1997, Appl. Opt., 36, 2107.

[8] Mio, C., Gong, T.,Terray, T., and Marr, D. W. M., 2000, Rev. Sci. Instr., 71, 2196.

[9] Ogura, Y., Kagawa, K., and Tanida, J., 2001, Appl. Opt., 40, 5430.

[10] Reicherter, M., Haist, T., Wagemann, E., and Tiziani, H., 1999, Opt. Lett., 24, 608.

[11] Curtis, J., Koss, A., and Grier, D., 2002, Opt. Commun., 207, 169.

[12] Kobayashi, Y., Igasaki, Y., Yoshida, N., Fukuchi, N., Toyoda, H., Hara, T., and Wu, M. H., 2000, Proc. SPIE, 3951, 158.

[13] Dufresne, E. R., and Grier, D. G., 1998, Rev. Sci. Instr., 69, 1974.

[14] Eriksen, R. L., Daria, V., and Glǘchstad, J., 2002, Opt. Express, 10, 597.

[15] Eriksen, R. L., Mogensen, P. C., and Glückstad, J., 2002, Opt. Lett., 27, 267.

[16] Mogensen, P. C., and Glückstad, J., 2000, Opt. Commun., 175, 75.

[17] Glückstad, J., and Mogensen, P. C., 2001, Appl. Opt., 40, 268.

[18] Zernike, F., 1955, Science, 121, 345.

[19] Glückstad, J., 1996, Opt. Commun., 130, 225. 
[20] Glückstad, J., 2000, US patent no. 6,011,874.

[21] Glüchstad, J., and Mogensen, P. C., 2000, Opt. Commun., 173, 169.

[22] Arlt, J., and Padgett, M., 2000, Opt. Lett., 25, 191.

[23] MacDonald, K., Paterson, L., Arlt, J., Sibett, W., and Dholakia, K., 2001, Opt. Lett., 26, 863.

[24] O’Neil, A., and Padgett, M., 2002, Opt. Lett., 27, 743. 